\title{
Osilodrostat for Cushing Disease and Its Role in Pediatrics
}

\author{
Urh Groselja, b, c Jaka Sikonja ${ }^{a}$ Tadej Battelino ${ }^{a, b}$ \\ ${ }^{a}$ Faculty of Medicine, University of Ljubljana, Ljubljana, Slovenia; ${ }^{b}$ Department of Endocrinology, Diabetes, and \\ Metabolic Diseases, University Children's Hospital, University Medical Center Ljubljana, Ljubljana, Slovenia; \\ 'Department of Cardiovascular Medicine, Stanford University, Palo Alto, CA, USA
}

\section{Keywords \\ Osilodrostat $\cdot$ Isturisa ${ }^{\circledR}$. Cushing disease $\cdot$ Pediatric · \\ Therapy}

\begin{abstract}
Background: Cushing disease (CD) is a very rare form of hypercortisolism caused by an adrenocorticotropic hormonesecreting pituitary adenoma. Clinical manifestations of CD can include central fat accumulation, arterial hypertension, glucose intolerance, skin atrophy with striae, and hypogonadism. Children are frequently diagnosed due to a growth stunt and excessive weight gain while classic cushingoid signs might be initially absent. Other children-specific presentations of CD are early or delayed puberty and hyperandrogenism in girls. Summary: We present the main outcomes of clinical trials of osilodrostat (Isturisa ${ }^{\circledR}$, Recordati) for $C D$, and its initial development as an aldosterone synthase inhibitor. Osilodrostat is indicated only when the surgical therapy of the pituitary adenoma is not an option or has not been curative; additionally, other steroidogenesis inhibitors were briefly summarized. Clinical trials of osilodrostat in children are lacking and we describe its potential role in the pediatric population. Key Messages: Osilodrostat is the first
\end{abstract}

adrenal steroidogenesis inhibitor to be European Medicines Agency- and United States Food and Drug Administrationapproved (both in 2020) for the treatment of adults with Cushing syndrome/disease. Phase II and III clinical trials have shown its efficacy in normalizing 24-h urinary-free cortisol and a good safety profile. Osilodrostat's pharmacological properties and safety are currently being evaluated in a small Phase II trial (NCT03708900) - the first trial in the pediatric population ( $<18$ years) with an estimated completion date in the year 2023.

(c) 2022 S. Karger AG, Basel

\section{Introduction}

Cushing disease (CD) is a very rare form of hypercortisolism caused by an adrenocorticotropic hormone (ACTH)-secreting pituitary adenoma $[1,2]$. Cushing syndrome (CS) is a wider entity that results from an excessive exposure to endogenous or exogenous glucocorticoids. Endogenous production of glucocorticoids can be a result of CD, an ectopic ACTH production by a neuroendocrine tumor or by cortisol-producing tumors of the adrenal glands [3]. It most commonly occurs in 
young- to mid-adulthood women and is an ultrarare disease with an incidence of 2-5 cases per 1 million people per year [4]. Clinical manifestations of CD can include various manifestations, such as central fat accumulation and obesity, arterial hypertension, glucose intolerance, striae, skin atrophy and fungal infections, osteopenia, hypogonadism, and depression $[1,2]$. In childhood, CD is even much rarer than in adults and is considered an ultrarare disease - only around $10 \%$ of all CD cases are diagnosed in children. While boys and girls are equally affected before and during puberty, girls are more frequently affected after puberty [5]. The classic cushingoid signs/ symptoms of CD are usually not the initial presentation seen in children. Most commonly, CD in children manifests with the combination of excessive weight gain and growth failure [6]. Other CD signs, such as early or delayed puberty, and/or signs of hyperandrogenism in girls could be present.

Accurate diagnosis of $\mathrm{CD}$ and appropriate treatment selection are warranted for optimal outcomes $[1,2]$. Initially, it is important to distinguish ACTH-dependent (CD) from ACTH-independent causes of CS. Transsphenoidal adenomectomy is considered as first-line therapy for $\mathrm{CD}$, enabling remission in up to $80 \% \mathrm{CD}$ patients. It is also a preferred surgical procedure in children or adolescents with CD [2]. Recurrence rates after the procedure are reported to be in the range of 5-35\%, with half of them in the first 5 years. Lifelong monitoring is thus required. In this case, repeated transsphenoidal surgery is recommended mostly in cases where tumor is evident on an MRI scan [2].

In cases of persistent or recurrent $\mathrm{CD}$, when the surgery is contraindicated or refused, or in combination with radiation therapy, medical therapy is indicated to treat hypercortisolism [2]. Adrenal steroidogenesis inhibitors are used as therapeutic agents for $\mathrm{CD}$, inhibiting adrenal enzymes to decrease glucocorticoid and/or adrenal androgen production, but do not target the ACTH-secreting pituitary adenoma. This group includes ketoconazole, metyrapone, mitotane, etomidate, and the recently approved osilodrostat. Other medical therapies are used in cases where adrenal steroidogenesis inhibitors are contraindicated or ineffective. They include pituitary-targeted drugs (cabergoline or pasireotide) or glucocorticoid receptor antagonists (mifepristone) $[2,4,7,8]$. Unfortunately, there is a lack of trials with direct comparisons of various medical therapies for $\mathrm{CD}$ and on the use of combinations of medical therapies [9]. The main medical therapies for CD are further presented in Table 1.
Osilodrostat (product code LCI699) is the last among the adrenal steroidogenesis inhibitors that were introduced for medical therapy of CD. Initially, Phase II clinical trials of osilodrostat were conducted for the treatment of hypertension and primary aldosteronism. However, in 2013, Novartis decided to discontinue its development in these indications and to repurpose the drug for the treatment of CD/CS [10]. After completion of clinical trials, in January 2020, European Medicines Agency approved osilodrostat for the treatment of "endogenous CS" in adults [11]. In March 2020, the United States Food and Drug Administration approved osilodrostat for the treatment of CD patients who either cannot undergo pituitary surgery or have undergone pituitary surgery but still have the disease [12]. Prior to that, in 2014, orphan designation $(\mathrm{EU} / 3 / 14 / 1345)$ was granted for osilodrostat for the treatment of CS [13]. Currently, osilodrostat is not approved for pediatric patients with CD. Nonetheless, a pediatric Phase II clinical trial is ongoing with a completion target set for 2023. In this focused review, we aimed to present the main characteristics of the osilodrostat as a therapy for CD, its development timeline and to briefly assess its possible future role in pediatric patients with $\mathrm{CD}$.

\section{Osilodrostat Clinical Trials}

\section{Phase I/II}

Osilodrostat was initially developed as a selective aldosterone synthase inhibitor that could offer a new approach in the treatment of hypertension, primary aldosteronism, and other conditions with a stimulated renin-angiotensin-aldosterone system. The timeline of osilodrostat development and approval is available in the online supplementary material (for all online suppl. material, see www.karger.com/doi/10.1159/000522054). In 2008 and 2009, four Phase II trials of osilodrostat were conducted: three for the treatment of hypertension and one for primary aldosteronism.

The clinical trial of primary aldosteronism (NCT00732771) found that low osilodrostat doses (1-2 mg/ day) were sufficient at inhibiting aldosterone synthesis and correcting hypokalemia. However, they only mildly reduced blood pressure [14]. In patients with essential hypertension (NCT00758524), different doses (0.25-1 mg/ day) of osilodrostat significantly lowered 24 -h ambulatory blood pressure compared to a placebo at the end of an 8-week randomized double-blind, double-dummy clinical trial. Blood pressure reductions were similar between osilodrostat $1 \mathrm{mg}$ /day and eplerenone $50 \mathrm{mg}$ twice 


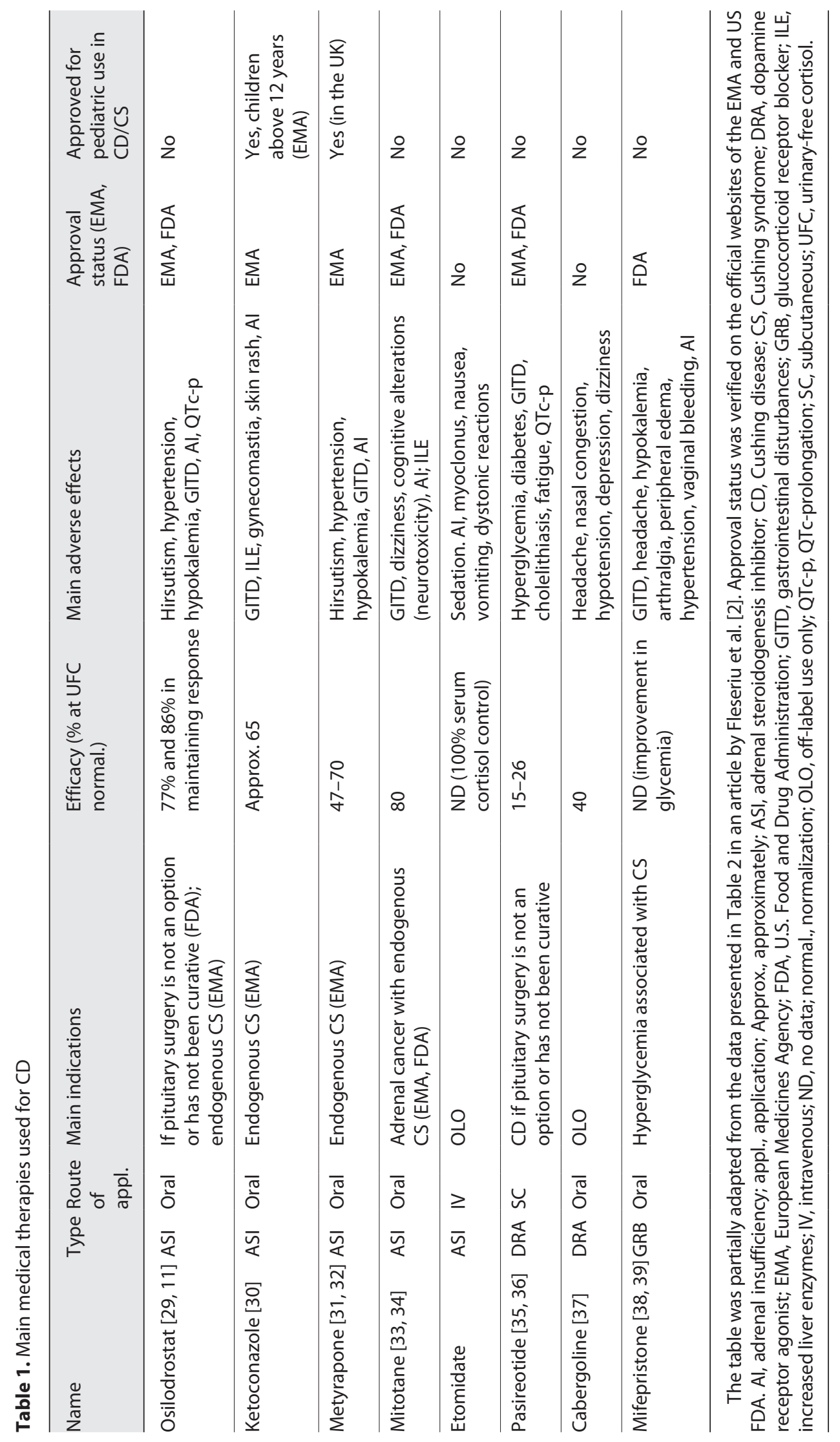


daily. ACTH stimulation test revealed that $20 \%$ of patients receiving osilodrostat $1 \mathrm{mg}$ daily had a blunted cortisol response [15]. The results of the latter study were confirmed by another clinical trial (NCT00817414) in 63 patients with hypertension. This proved the nonselectivity of osilodrostat for aldosterone synthase as a dose-dependent inhibition was observed at an ACTH stimulation test [16]. Moreover, osilodrostat as an add-on therapy, administered in 0.5-2-mg doses, was not associated with statistically significant blood pressure reductions when compared to a placebo in a study of patients with resistant hypertension (NCT00817635) [17]. All four clinical trials showed that osilodrostat is well tolerated in patients, but had a modest blood pressure-lowering effect, probably due to an accumulation of aldosterone precursors. Additionally, higher doses led to an impaired cortisol production. These findings drove the repurposing of osilodrostat away from the treatment of hypertension and to the treatment of endogenous hypercortisolism [18].

After repurposing, the first proof of concept clinical trial for the treatment of CD (NCT01331239) was started in 2011. It consisted of two parts: LINC-1 (LCI699 IN Cushing-1) and LINC-2. Thirty-one patients with CD and urinary-free cortisol (UFC) levels $>1.5$ upper limit of normal (ULN) were enrolled and introduced to osilodrostat for 10 weeks (LINC-1) and 22 weeks (LINC-2).

Twelve patients included in LINC-1 started with $4 \mathrm{mg} /$ day of osilodrostat. The dose increased every 2 weeks according to UFC. The primary endpoint was defined as $\mathrm{UFC} \leq \mathrm{ULN}$ or $\geq 50 \%$ UFC reduction from baseline levels at week 10. All 12 patients had a $\geq 50 \%$ UFC reduction from baseline, while 11 patients $(92 \%)$ had UFC $\leq$ ULN at week 10, thus proving the concept and demonstrating the efficacy of osilodrostat in lowering cortisol levels. UFC rose above ULN 2 weeks after discontinuation of osilodrostat [19].

LINC-2 trial enrolled 19 patients, four of whom had been included in LINC-1 and were reenrolled if UFC $>$ ULN. Osilodrostat was introduced at the maximum efficacious and tolerable dose from LINC-1 for reenrolled patients and at $4 \mathrm{mg} /$ day for osilodrostat-naive patients (or $10 \mathrm{mg} /$ day if baseline UFC $>3 \times \mathrm{ULN}$ ). Dosage was increased every 2 weeks if UFC remained below ULN. LINC-2 confirmed the findings from LINC-1, since UFC decreased from baseline and normal UFC levels were observed in all patients. At week 22, 15/17 patients had UFC $<\mathrm{ULN}$ at week 22 and two had experienced a roughly $50 \%$ decrease of UFC. One patient discontinued the trial due to an administrative reason and one due to an osilodrostat-related papular rash [20]. Another Phase II trial
(NCT02468193) evaluated the efficacy and safety of osilodrostat in a small cohort $(N=9)$ of patients with CS except $\mathrm{CD}$ in short (up to 12 weeks) and long term (up to 48 weeks). It showed similar results to LINC-1 and LINC-2 that only enrolled patients with CD [21].

Two Phase I trials were conducted for osilodrostat in a single $30 \mathrm{mg}$ dose. The first (NCT02399202) found no significant differences in exposure to osilodrostat between individuals with normal kidney function, severe function impairment, and end-stage renal disease [11, $22]$. On the other hand, hepatic function impairment had a significant effect on its pharmacokinetics (NCT02372084). Patients with severe (Child-Pugh C) and moderate (Child-Pugh B) hepatic impairment had higher systemic exposure (AUC 1.4- and 2.7-times higher) and osilodrostat half-life was increased by 1.8 - and 3.7-times compared to individuals with normal liver function. No significant differences in exposure parameters were seen at a Child-Pugh A stage $[11,23]$.

\section{Phase III/IV}

LINC-3 and LINC-4 trials (NCT02180217 and NCT02697734) included 137 and 73 adults with CD that had UFC $>1.5 \times$ ULN and elevated morning ACTH levels (LINC-3) or UFC $>1.3$ ULN (LINC-4). LINC-3 was the first study that included a double-blind placebo-controlled withdrawal phase. It showed that $86 \%$ of patients on a fixed osilodrostat dose (mean dose $10 \mathrm{mg} /$ day) maintained a complete response (UFC $\leq \mathrm{ULN}$ ) during 8 weeks compared to only $29 \%$ on placebo. All patients included in this phase were complete responders before randomization to osilodrostat or placebo [24]. In the second Phase III study LINC-4, patients enrolled started a 12week double-blind placebo-controlled phase with dose adjustments according to efficacy and tolerability. In the osilodrostat group, $77 \%$ of individuals achieved UFC $\leq \mathrm{ULN}$ while only $8 \%$ in the placebo group at the end of this phase. Osilodrostat recipients maintained the response until week 36 [25]. Moreover, patients on osilodrostat experienced improvements in clinical parameters of hypercortisolism: reduction of body weight, blood pressure, cholesterol, fasting glucose, and $\mathrm{HbA} 1 \mathrm{c}$ concentrations, along with an improved quality of life and psychological state [24].

\section{Ongoing Clinical Trials}

Currently, two ongoing Phase II clinical trials (NCT03606408 and NCT03708900) are registered at clinicaltrials.gov. The first is an open-label study and aims to investigate long-term adverse events and clinical 


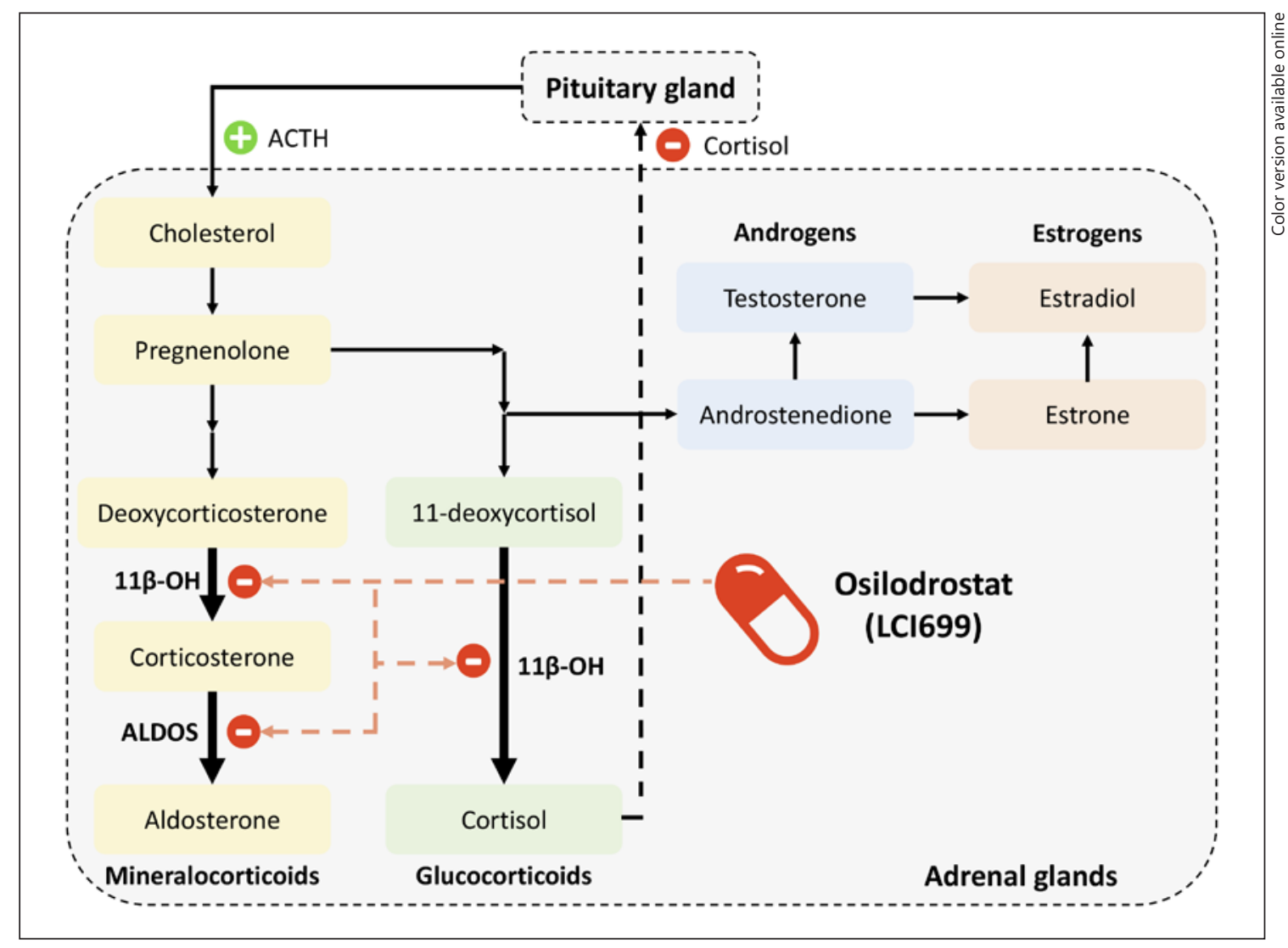

Fig. 1. Mechanism of action of osilodrostat (LCI699). Osilodrostat blocks the last enzymes in both glucocorticoid and mineralocorticoid synthesis pathways, thereby halting the production of both cortisol and aldosterone. The pituitary responds by an increased production of ACTH to low cortisol levels. By blocking ALDOS and $11 \beta-\mathrm{OH}$, upstream metabolites, including androgens, start to accumulate in the cortex of adrenal glands [10]. ACTH, adrenocorticotropic hormone; $11 \beta-O H, 11 \beta$-hydroxylase; ALDOS, aldosterone synthase.

benefits in adult patients with endogenous CS that were included in a previously completed osilodrostat trial and benefited from the drug. All included patients will be receiving the predetermined efficacious osilodrostat dose in a prior trial.

The other ongoing trial (NCT03708900) will be the first to include a small cohort of pediatric patients $(<18$ years) with CD. Its goal is to evaluate the pharmacokinetics, pharmacodynamics, and tolerability of osilodrostat. The expected completion date is in 2023.

In summary, osilodrostat proved to be an efficacious treatment for CS and CD. Due to its oral administration and good tolerance, osilodrostat could help patients suffering from hypercortisolism. Nonetheless, to prevent adverse events, they should be regularly monitored for timely dose adjustments.

Osilodrostat for Cushing Disease

\section{Pharmacology}

\section{Mechanisms of Action}

The production of corticosteroids in the adrenal glands is closely regulated by two endocrine systems: the reninangiotensin-aldosterone system and the hypothalamuspituitary-adrenal axis. Aldosterone and cortisol, the main mineralocorticoid and glucocorticoid hormone, are produced from cholesterol and share some of the same precursors (shown in Fig. 1). An enzyme 11 $\beta$-hydroxylase (CYP11B1) catalyzes the last step in the synthesis of cortisol while CYP11B2, also known as aldosterone synthase, is responsible for the conversion of deoxycorticosterone to aldosterone [18]. While osilodrostat inhibits both enzymes, it has a greater potency for inhibition of CYP11B1 [26], thereby reducing the synthesis of aldosterone and cortisol. Low cortisol levels stimulate the hypothalamus- 
pituitary-adrenal axis and ACTH from the pituitary which in the end stimulates steroidogenesis and the production of glucocorticoids, mineralocorticoids, and adrenal androgens [18].

\section{Pharmacokinetics}

Osilodrostat is designed to be taken orally due to its rapid absorption $\left(t_{\max } \cong 1 \mathrm{~h}\right)$ in the gastrointestinal tract and minor first-pass metabolism. Administration of osilodrostat with food slightly reduces its bioavailability ( $11 \%$ reduction of AUC compared to a fasting state). It has a high volume $(\approx 100 \mathrm{~L})$ of distribution, which indicates good penetration into tissues where no significant accumulation is observed. The majority of osilodrostat is metabolized by cytochromes P450 and UDP-glucuronosyltransferases and excreted by kidneys. Only around $20 \%$ of the dose is excreted in the unchanged form [11, 27].

\section{Adverse Effects, Drug Interactions, and Use in Pregnancy and during Lactation}

Osilodrostat proved to be tolerable by patients in the completed Phase II and III trials; however, a minority of patients discontinued participation in the studies due to an osilodrostat-related adverse event which were in general mild and nonspecific. The most frequent by relative frequency in the largest Phase III trial (LINC-3) that included 137 patients were nausea (42\%), headache (34\%), fatigue (28\%), and adrenal insufficiency (28\%). In the randomized double-blind withdrawal phase, the overall frequency of adverse events was similar in the osilodrostat-receiving group and placebo-receiving group. Hypocortisolism and related adverse events occurred in 70\% and $42 \%$ of patients, most commonly observed in the dose titration phase. They were manageable by dose adjustments or glucocorticoid supplementation [24, 28]. Patients taking osilodrostat in the double-blind placebocontrolled LINC-4 trial reported decreased appetite (38\% vs. $16 \%$ placebo), arthralgia ( $35 \%$ vs. $8 \%$ ), nausea ( $31 \%$ vs. $12 \%)$, hypocortisolism-related adverse events (15\% vs. $0 \%$ ), all of which were mild (grade 1-2) and manageable as previously mentioned [25].

Aldosterone and cortisol production inhibition by osilodrostat can result in a cortisol withdrawal syndrome or adrenal insufficiency. It is therefore advised to monitor serum cortisol in patients taking osilodrostat and intervene with dose reductions or glucocorticoid supplementation if cortisol levels fall below the lower limit of normal [11]. Hypokalemia and hypertension can occur as a consequence of the accumulation of biologically active aldo- sterone precursors. With this in mind, potassium levels should be determined prior to the introduction of osilodrostat and in regular intervals afterwards $[9,11]$. The follow-up of these patients should also include an electrocardiogram since a dose-dependent QTc interval prolongation has been reported in patients taking osilodrostat $[11,27]$. Female patients in the LINC-3 trial had adverse events related to androgen and estrogen increase and reported mild hirsutism (11\%) and acne (11\%) [24].

Osilodrostat should be cautiously used with drugs that cause QTc-prolongation, such as certain antibiotics and antidepressants, and hypokalemia. Prior to excretion, the majority of the drug is metabolized by cytochromes $\mathrm{P} 450$. Furthermore, certain cytochromes can be inhibited or induced by osilodrostat. Thus, a clinician prescribing osilodrostat should be cautious at its initiation or at introducing a new drug in a patient already receiving osilodrostat [11].

Preclinical data in animals indicated that osilodrostat could have a toxic effect on the fetus and should not be prescribed to pregnant women or to women in the childbearing period who are not using contraception. Due to a lack of data on the effect of osilodrostat on newborns/ infants, it is also advised to avoid breastfeeding for at least 1 week after discontinuing treatment [11].

\section{Dosage and Administration}

Osilodrostat comes in the form of film-coated tablets for oral use and can be taken with or without food. The starting dose for adults with CS is $2 \mathrm{mg}$ daily and is gradually titrated (maximum dose $30 \mathrm{mg}$ twice daily) to achieve UFC $<$ ULN while taking the patient's tolerability into account. Dose adjustments should be made in patients with severe (Child-Pugh C) and moderate (ChildPugh B) liver impairment, but not for those with kidney function impairment. Response to the drug should be monitored every 1-2 weeks with 24-h UFC and serum cortisol measurements [11].

\section{Potential Role of Osilodrostat for Pediatric Patients}

$\mathrm{CD}$ is extremely rare in children and adolescents, which is also reflected in scarce data on clinical management and the lack of clinical trials available on pediatric CD. Transsphenoidal adenomectomy is considered a first-line therapy for $\mathrm{CD}$ in children and adolescents. If $\mathrm{CD}$ persists or recurs, or when the surgery is contraindicated or refused, medical therapy is indicated [2]. Adrenal steroidogenesis inhibitors, mostly ketoconazole and me- 
tyrapone (but not pasireotide), are the first-line medical therapy for pediatric CD [2]. Osilodrostat has not yet been approved for pediatric patients but is currently being evaluated in a small Phase II trial (NCT03708900), which is expected to be completed in 2023. However, most of the current medical therapies for CD in children and adolescents were not tested in pediatric patients and are currently used off-label. Based on current evidence indicating a superior efficacy and safety profile of osilodrostat over other adrenal steroidogenesis inhibitors, it might already be considered for off-label use in selected pediatric patients. However, we find it reasonable that pediatric patients with $\mathrm{CD}$ that are candidates for medical therapy participate in the ongoing clinical trial on pediatric patients. In addition, children with $C D$ should be referred to multidisciplinary tertiary centers with pediatric endocrinologists, experts in managing disorders of the pituitary, and with specialized neurosurgery units [2].

\section{Conclusions}

Osilodrostat is the first adrenal steroidogenesis inhibitor to be European Medicines Agency- and United States Food and Drug Administration-approved (both in 2020) for the treatment of adults with $\mathrm{CD}$, when surgical resection of the pituitary adenoma is not indicated, desired, or effective. Thus far, other steroidogenesis inhibitors have been used off-label in pediatric patients for this indica- tion. Phase II and III clinical trials have shown osilodrostat's efficacy in normalizing the 24-h UFC and a good safety profile $[19,20,24,25]$. Osilodrostat has not yet been approved for pediatric patients, but is currently being evaluated in a small Phase II trial (NCT03708900), which is expected to bring more answers on its pharmacological properties and safety.

\section{Conflict of Interest Statement}

T.B. (PI) and U.G. (sub-I) are currently involved in a pediatric trial of osilodrostat (NCT03708900). J.S. declares no conflicts of interest.

\section{Funding Sources}

The work was partly funded by the Slovenian Research Agency program P3-0434. The authors confirm independence from the sponsors. The sponsor had no role in study design, in the collection, analysis, and interpretation of data, in the writing of the report, and in the decision to submit the article for publication.

\section{Author Contributions}

Urh Groselj: investigation, conceptualization, and writing original draft; Jaka Sikonja: investigation, writing - original draft, and visualization; and Tadej Battelino: investigation and writing - original draft.

\section{References}

1 Melmed S. Pituitary-tumor endocrinopathies. N Engl J Med. 2020 Mar;382(10):93750.

2 Fleseriu M, Auchus R, Bancos I, Ben-Shlomo A, Bertherat J, Biermasz NR, et al. Consensus on diagnosis and management of Cushing's disease: a guideline update. Lancet Diabetes Endocrinol. 2021 Dec;9(12):847-75.

3 Creemers SG, Feelders RA, de Jong FH, Franssen GJH, de Rijke YB, van Koetsveld PM, et al. Osilodrostat is a potential novel steroidogenesis inhibitor for the treatment of cushing syndrome: an in vitro study. J Clin Endocrinol Metab. 2019 Aug;104(8):3437-49.

4 Nieman LK, Biller BMK, Findling JW, Murad $\mathrm{MH}$, Newell-Price J, Savage MO, et al. Treatment of cushing's syndrome: an endocrine society clinical practice guideline. J Clin Endocrinol Metab. 2015 Aug;100(8):2807-31.

5 Libuit LG, Karageorgiadis AS, Sinaii N, Nguyen May NM, Keil MF, Lodish MB, et al. A gender-dependent analysis of Cushing's disease in childhood: pre- and postoperative followup. Clin Endocrinol. 2015 Jul;83(1):72-7.

6 Storr HL, Alexandraki KI, Martin L, Isidori AM, Kaltsas GA, Monson JP, et al. Comparisons in the epidemiology, diagnostic features and cure rate by transsphenoidal surgery between paediatric and adult-onset Cushing's disease. Eur J Endocrinol. 2011 May;164(5):667-74.

7 Tritos NA, Biller BMK. Current management of Cushing's disease. J Intern Med. 2019 Nov; 286(5):526-41.

8 Theodoropoulou M, Reincke M. Tumor-directed therapeutic targets in cushing disease. J Clin Endocrinol Metab. 2019 Mar;104(3): 925-33.

9 Pivonello R, Ferrigno R, De Martino MC, Simeoli C, Di Paola N, Pivonello C, et al. Medical treatment of cushing's disease: an overview of the current and recent clinical trials. Front Endocrinol. 2020 Dec;11:648.

10 Duggan S. Osilodrostat: first approval. Drugs. 2020 Apr;80(5):495-500.
11 European Medicines Agency. Osilodrostat (Isturisa ${ }^{\varpi}$ ): summary of product characteristics; 2020. Available from: https://www.ema. europa.eu/en/documents/product-information/isturisa-epar-product-information_en. pdf.

12 United States Food and Drug Administration. FDA approves new treatment for adults with cushing's disease; 2021. Available from: https: //www.fda.gov/news-events/press-announcements/fda-approves-new-treatmentadults-cushings-disease.

13 European Medicines Agency. EU/3/14/1345 orphan designation for the treatment of Cushing's syndrome; 2021. Available from: https: //www.ema.europa.eu/en/medicines/ human/orphan-designations/eu3141345.

14 Amar L, Azizi M, Menard J, Peyrard S, Watson C, Plouin PF. Aldosterone synthase inhibition with LCI699: a proof-of-concept study in patients with primary aldosteronism. Hypertension. 2010 Nov;56(5):831-8. 
15 Calhoun DA, White WB, Krum H, Guo W, Bermann G, Trapani A, et al. Effects of a novel aldosterone synthase inhibitor for treatment of primary hypertension: results of a randomized, double-blind, placebo- and active-controlled phase 2 trial. Circulation. 2011 Nov;124(18):1945-55.

16 Andersen K, Hartman D, Peppard T, Hermann D, Van Ess P, Lefkowitz M, et al. The effects of aldosterone synthase inhibition on aldosterone and cortisol in patients with hypertension: a phase II, randomized, doubleblind, placebo-controlled, multicenter study. J Clin Hypertens. 2012 Sep;14(9):580-7.

17 Karns AD, Bral JM, Hartman D, Peppard T, Schumacher C. Study of aldosterone synthase inhibition as an add-on therapy in resistant hypertension. J Clin Hypertens. 2013 Mar; 15(3):186-92.

18 Schumacher CD, Steele RE, Brunner HR. Aldosterone synthase inhibition for the treatment of hypertension and the derived mechanistic requirements for a new therapeutic strategy. J Hypertens. 2013 Oct;31(10):2085-93.

19 Bertagna X, Pivonello R, Fleseriu M, Zhang Y, Robinson P, Taylor A, et al. LCI699, a potent $11 \beta$-hydroxylase inhibitor, normalizes urinary cortisol in patients with cushing's disease: results from a multicenter, proof-ofconcept study. J Clin Endocrinol Metab. 2014 Apr;99(4):1375-83.

20 Fleseriu M, Pivonello R, Young J, Hamrahian $\mathrm{AH}$, Molitch ME, Shimizu C, et al. Osilodrostat, a potent oral $11 \beta$-hydroxylase inhibitor: 22-week, prospective, phase II study in Cushing's disease. Pituitary. 2016 Apr;19(2):13848.

21 Novartis. Basel: a Phase II, open-label, dose titration, multi-center study to assess the safety/tolerability and efficacy of osilodrostat in patients with all types of endogenous Cushing's syndrome except Cushing's disease technical result summary; 2021. Available from: https://www.novctrd.com/ctrdweb/trialresult/trialresults/pdf?trialResultId $=17549$.

22 Novartis. Basel: a Phase I, open-label, multicenter, single dose, parallel group study to evaluate the pharmacokinetics and safety of LCI699 in subjects with varying degrees of impaired renal function compared to subjects with normal renal function - technical result summary; 2021. Available from: https://www. novctrd.com/ctrdweb/trialresult/trialresults/ pdf?trialResultId $=16047$.

23 Novartis. Basel: a Phase I, open-label, multicenter, single dose, parallel group study to evaluate the pharmacokinetics and safety of LCI699 in subjects with impaired hepatic function compared to subjects with normal hepatic function - technical result summary; 2021. Available from: https://www.novctrd. com/\#/product?type=clinicalP\& medicalConditionId=201\&productId $=166$.

24 Pivonello R, Fleseriu M, Newell-Price J, Bertagna X, Findling J, Shimatsu A, et al. Efficacy and safety of osilodrostat in patients with Cushing's disease (LINC 3): a multicentre phase III study with a double-blind, randomised withdrawal phase. Lancet Diabetes Endocrinol. 2020 Sep;8(9):748-61.

25 Gadelha M, Bex M, Feelders RA, Heaney AP, Auchus RJ, Gilis-Januszewska A, et al. Osilodrostat is an effective and well-tolerated treatment for Cushing's disease (CD): results from a phase III study with an upfront, randomized, double-blind, placebo-controlled phase (LINC 4). Endocrine Abstracts. 2021.

26 Meredith EL, Ksander G, Monovich LG, Papillon JPN, Liu Q, Miranda K, et al. Discovery and in vivo evaluation of potent dual CYP11B2 (aldosterone synthase) and CYP11B1 inhibitors. ACS Med Chem Lett. 2013 Dec; 4(12):1203-7.

27 Novartis. Basel: a Phase III, multi-center, randomized, double-blind, 48 week study with an initial 12 week placebo-controlled period to evaluate the safety and efficacy of osilodrostat in patients with Cushing's disease - clinical trial protocol; 2021. Available from: https:// clinicaltrials.gov/ct2/show/NCT02697734.

28 Dougherty JA, Desai DS, Herrera JB. Osilodrostat: a novel steroidogenesis inhibitor to treat Cushing's disease. Ann Pharmacother. 2021 Aug;55(8):1050-60.

29 United States Food and Drug Administration. Isturisa (osilodrostat): highlights of prescribing information; 2021. Available from: https: //www.accessdata.fda.gov/drugsatfda_docs/ label/2020/212801s000lbl.pdf.

30 European Medicines Agency. Ketoconazole HRA $200 \mathrm{mg}$ tablets - summary of product characteristics; 2021. Available from: https:// www.ema.europa.eu/en/documents/product-information/ketoconazole-hra-eparproduct-information_en.pdf.

31 Varlamov EV, Han AJ, Fleseriu M. Updates in adrenal steroidogenesis inhibitors for Cushing's syndrome: a practical guide. Best Pract Res Clin Endocrinol Metab. 2021 Jan;35(1): 101490.

32 Datapharm. METOPIRONE $250 \mathrm{mg}$ : summary of product characteristics; 2021. Available from: https://www.medicines.org.uk/ emc/product/4092/smpc\#gref.

33 European Medicines Agency. Lysodren 500 mg tablets: summary of product characteristics; 2021. Available from: https://www.ema. europa.eu/en/documents/product-information/lysodren-epar-product-information en.pdf.

34 United States Food and Drug Administration. Lysodren (mitotane) tablets: highlights of prescribing information; 2021. Available from: https://www.accessdata.fda.gov/drugsatfda_docs/label/2021/016885s029lbl.pdf.

35 European Medicines Agency. Signifor 0.3 solution for injection: summary of product characteristics; 2021. Available from: https:// www.ema.europa.eu/en/documents/product-information/signifor-epar-product-information_en.pdf.

36 United States Food and Drug Administration. SIGNIFOR (pasireotide diaspartate) injection: highlights of prescribing information; 2021. Available from: https://www.accessda$\mathrm{t}$ a.f d a.gov/d r ugsatfd a label/2012/200677lbl.pdf.

37 United States Food and Drug Administration. application number: 212801 orig 1 s000 - summary review; 2021. Available from: https:// www.accessdata.fda.gov/drugsatfda_docs/ nda/2020/212801Orig1s000SumR.pdf.

38 European Medicines Agency. Corluxin: withdrawal of the marketing authorisation application; 2021. Available from: https://www. ema.europa.eu/en/medicines/human/withdrawn-applications/corluxin.

39 United States Food and Drug Administration. Korlym $^{\text {Tw }}$ (mifepristone) 300 mg tablets: highlights of prescribing information; 2021. Available from: https://www.accessdata.fda.gov/ drugsatfda_docs/label/2012/202107s000lbl. pdf. 